\title{
Recent advances in 2D hexagonal boron nitride (2D-hBN) applied as the basis of electrochemical sensing platforms
}

\author{
Alejandro García-Miranda Ferrari ${ }^{1}$ - Samuel J. Rowley-Neale ${ }^{1}$ • Craig E. Banks ${ }^{1}$ \\ Received: 18 October 2020 / Accepted: 12 November 2020 / Published online: 7 December 2020 \\ (C) The Author(s) 2020
}

\begin{abstract}
$2 \mathrm{D}$ hexagonal boron nitride $(2 \mathrm{D}-\mathrm{hBN})$ is a lesser utilised material than other $2 \mathrm{D}$ counterparts in electrochemistry due to initial reports of it being non-conductive. As we will demonstrate in this review, this common misconception is being challenged, and researchers are starting to utilise $2 \mathrm{D}-\mathrm{hBN}$ in the field of electrochemistry, particularly as the basis of electroanalytical sensing platforms. In this critical review, we overview the use of $2 \mathrm{D}-\mathrm{hBN}$ as an electroanalytical sensing platform summarising recent developments and trends and highlight future developments of this interesting, often overlooked, $2 \mathrm{D}$ material.
\end{abstract}

Keywords $2 \mathrm{D}$ hexagonal boron nitride $\cdot 2 \mathrm{D}-\mathrm{hBN} \cdot$ Electroanalysis $\cdot$ Sensors $\cdot$ Electrochemistry

\section{Introduction}

2D nanomaterials have attracted significant interest in a plethora of fields, particularly electrochemistry and electroanalysis, as can be observed by this article being part of this special issue. The first 2D nanomaterial that gained significant attraction and launched this field was graphene after Novoselov and Geim first isolated monolayer graphene and its interest has only grown since the award of their Nobel Prize in 2010, due to its reported extraordinary physical and chemical properties [1]. Nowadays, the term "2D nanomaterials" include more than a hundred different materials, including the family of carbon nanomaterials such as graphene, CNTs, fullerenes, graphene oxide, graphene QDs and other variants [2]. This has been rapidly extended to, for example, hexagonal boron nitride (2D-hBN, the so-called white graphene), transition metal chalcogenides (TMDs) (such as $\mathrm{MoS}_{2}$, $\mathrm{MoSe}_{2}, \mathrm{WS}_{2}, \mathrm{WSe}_{2}$ ) and homoatomic materials (such as antimonene, silicene, germanene, phosphorene) [3-11].

Published in the topical collection 2D Nanomaterials for Electroanalysis with guest editor Sabine Szunerits.

Craig E. Banks

c.banks@mmu.ac.uk

1 Faculty of Science and Engineering, Manchester Metropolitan University, Chester Street, Manchester M1 5GD, UK
A graphical representation of the exponential interest of research in graphene, 2D materials and boron nitride is depicted in Fig. 1, where the number of articles published between the years 2000 and 2020 is compared for the search terms "graphene", "2D material" and "boron nitride". It is clear upon inspection of Fig. 1 that the first isolation of pristine graphene in 2004, and then, the Nobel Prize Award in 2010 helped putting first graphene and then other 2D materials (including h-BN) in the spotlight of a wide variety of different research topics. To name a few, materials science, chemistry and physics are the main topics for graphene research, being energy, materials science and chemistry the main ones for both terms "2D materials" and "boron nitride".

The application of electrochemistry in analytical methods investigates changes in electrical properties that are related to chemical reactions/parameters. Electroanalysis has been widely explored for quality control [12], water [13] and environmental monitoring [14, 15], forensics [16-18], food [19-21] and biomedical [22, 23] applications to name just a few. In the application of 2D nanomaterials, they have reported to have significant benefits over other nanomaterials in the field of electroanalysis [8, 24]. Monolayered 2D materials offer high surface-to-volume ratios, which enhances their chemical reactivity than the one exhibited by their bulk form (inert activity) $[25,26]$. In this critical review, we overview the use of $2 \mathrm{D}-\mathrm{hBN}$ as the basis of electroanalytical sensing platforms and summarising recent exciting developments and highlight potential future developments of this interesting material. 


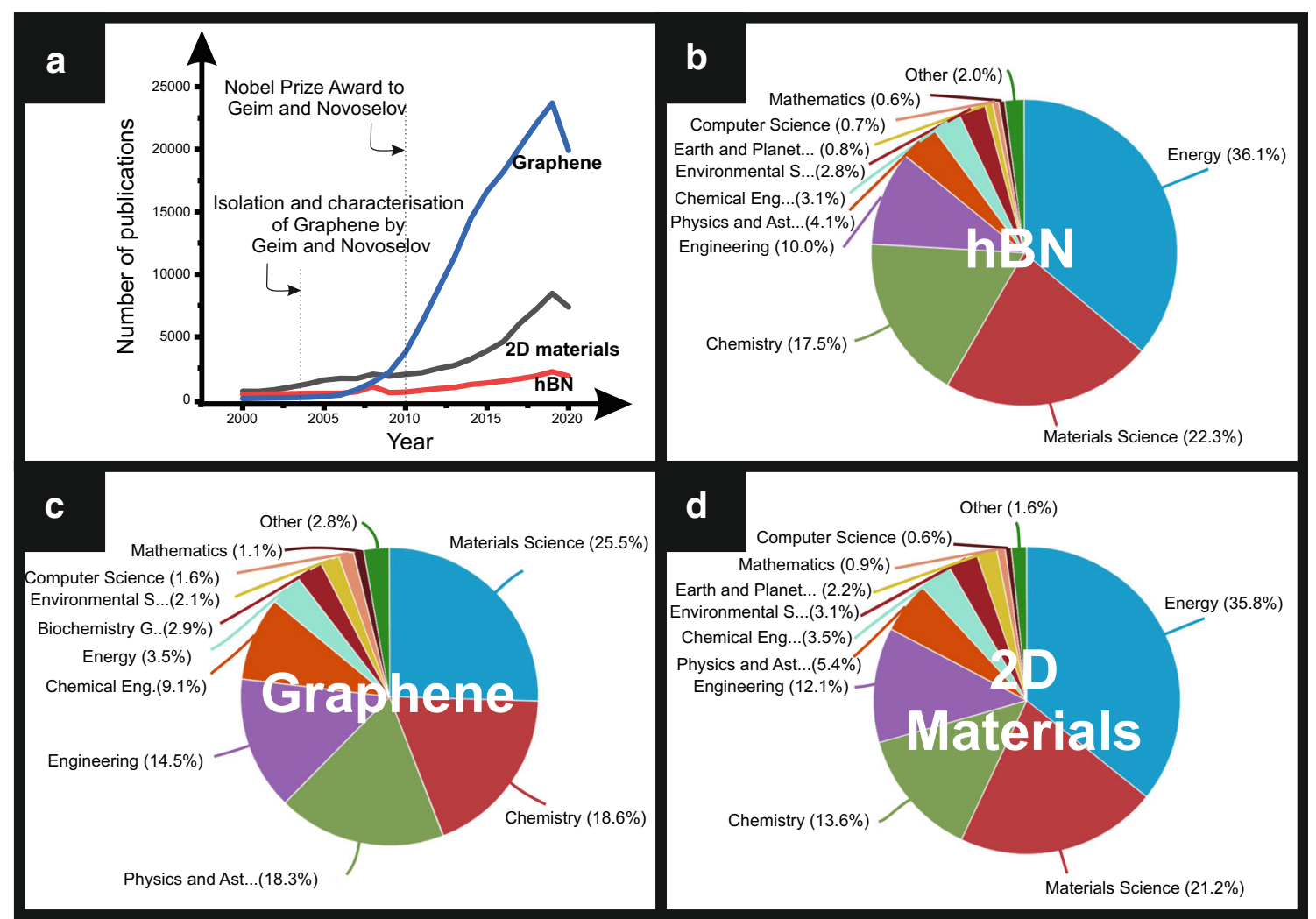

Fig. 1 Number of publications and related fields to "graphene", "2D materials" and "boron nitride" in the last 20 years. Data obtained from Scopus (accessed at the time of the submission of this paper)

\section{D-hexagonal boron nitride in electroanalysis}

2D-hexagonal boron nitride (2D-hBN) is a structural analogue of graphite which presents an $\mathrm{sp}^{2}$ hybridisation of $\mathrm{B}-\mathrm{N}$ bonds in a layered honeycomb structure comprising rings of borazine $\left(\mathrm{B}_{3} \mathrm{~N}_{3} \mathrm{H}_{6}\right)$ [27]. Boron nitride $(\mathrm{BN})$ is chemically stable exhibiting four well-known polymorphs: wurtzite, rhombohedral, cubic and hexagonal [28] (and references therein). Figure 2 overviews the various hexagonal boron nitride structures highlighting the intra- and inter-planar sizes and the edges of a nanosheet. Nanoribbons can be in either a zigzag (B- or N-edged) or armchair (BN pair edge) conformation, which are typically comprised of lateral sizes from a few hundred nanometres to tens of microns, depending on the various fabrication approaches employed. 2D-hBN layers can also stack on each other forming few- and multi-layers via van der Waals forces at a distance of $0.333 \mathrm{~nm}$ [27]. Other common structural forms are nanotubes, fullerenes and quantum dots.

Typically, 2D materials are fabricated via one of two routes: a bottom-up (BU) or a top-down (TD) method. TD approaches start from bulk materials as a starting point and transform it to achieve a monolayer. On the other hand, bottom-up (BU) approaches synthesise a pristine/monolayer from precursor(s). BU fabrication methods give larger yields of end products but do exhibit larger contamination (which might affect the electrochemistry of the material itself [31-33]), defects and lower quality of the nanosheets; on the contrary, TD lead to pristine materials but in lower quantities. In the case of hexagonal boron nitride, liquid-phase, ultrasonication-assisted, microwave-assisted, chemically assisted and mechano-chemical exfoliation methods are the most common BU approaches utilised within the literature [34-36]. In regard to BU methods, the most common are chemical vapour deposition (CVD) and physical vapour deposition (sputtering) [37-40]. Figure 3 includes a schematic representation of exfoliation (a), chemical vapour deposition (b) and sputtering (c) manufacturing methods for boron nitride from the literature [37, 41, 42]. The various fabrication approaches reported to $2 \mathrm{D}-\mathrm{hBN}$ will not be covered in detail here, but we note that the fabrication route can affect the electrochemical performance of 2D-hBN (see, for example, the work by Khan et al. reporting the effect of surfactants in the electrochemical properties of 2D-hBN [43]).

According to the literature, 2D-hBN has a wide band gap of ca. $5.6 \mathrm{eV}[44,45]$; therefore, it is usually reported as an electrical insulator $[45,46]$. Theoretical simulations have been used to describe physical and chemical properties of multiple combinations of synthesis, characterisation and substrates with 2D-hBN [47-49]. From the electroanalysis perspective, researchers are in a continuous search of new electrode materials that offer a wide useful potential range, low background 


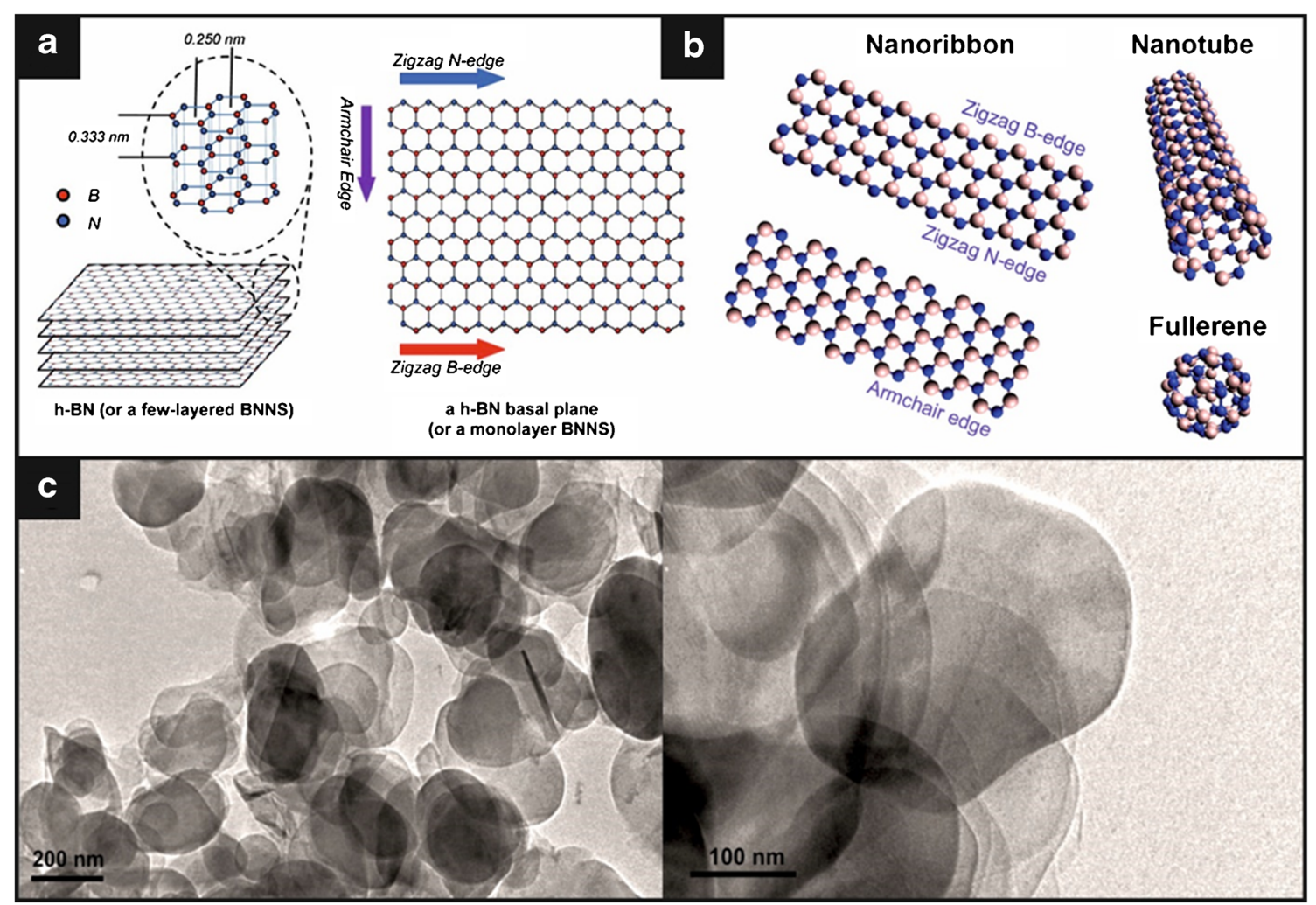

Fig. 2 An overview of hexagonal boron nitride structures. Figures reproduced from a [27], b [29] and $\mathbf{c}$ [30]

current, reproducibility and stability, and lastly fast electron transfer kinetics. Although $2 \mathrm{D}-\mathrm{hBN}$ is commonly reported as non-conductive $[49,50]$, it is finding use as the basis of electroanalytical sensing platforms. Table 1 provides a summary of some recent advances of 2D-hBN applied to electroanalytical applications, and one can readily see that there is a limited amount of literature reporting the use of 2D-hBN as the main active material for this purpose.

Uosaki et al. [49] first reported the use of 2D-hBN upon gold (single crystal) electrodes as an electrocatalyst for the important electrochemical reaction, the oxygen reduction reaction (ORR) which was only possible on the gold electrode with no electrocatalytic effect observed upon glassy carbon electrodes. While the ORR was found to proceed to produce hydrogen peroxide, rather than the desired product of water, this work was seminal in demonstrating that there is an importance of 2D-hBN-substrate interaction and that 2D-hBN can be utilised in electrochemistry [49]. This work has been extended by others (see, for example, references $[43,67]$ and [68]).

Following the above seminal work, Khan et al. reported for the first time the utilisation of $2 \mathrm{D}-\mathrm{hBN}$ as the basis of an electrochemical sensing platform using the example of the simultaneous electrochemical sensing of dopamine and uric acid determination via drop-casting the 2D-hBN onto screenprinted graphite macroelectrodes (SPEs) [30]. The electrochemical response was found to be highly dependent upon the interaction of the 2D-hBN and the underlying supporting electrode material and amount of deposited material, giving an electrocatalytic response in comparison to the (bare)

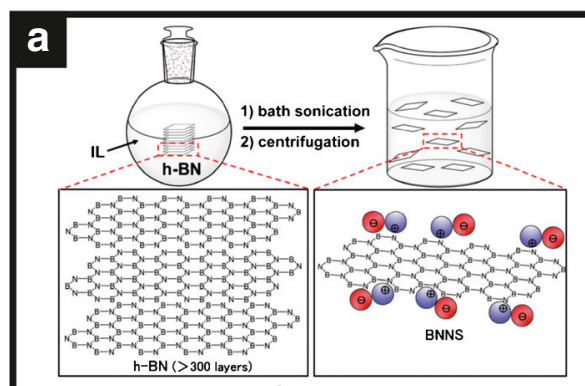

Exfoliation
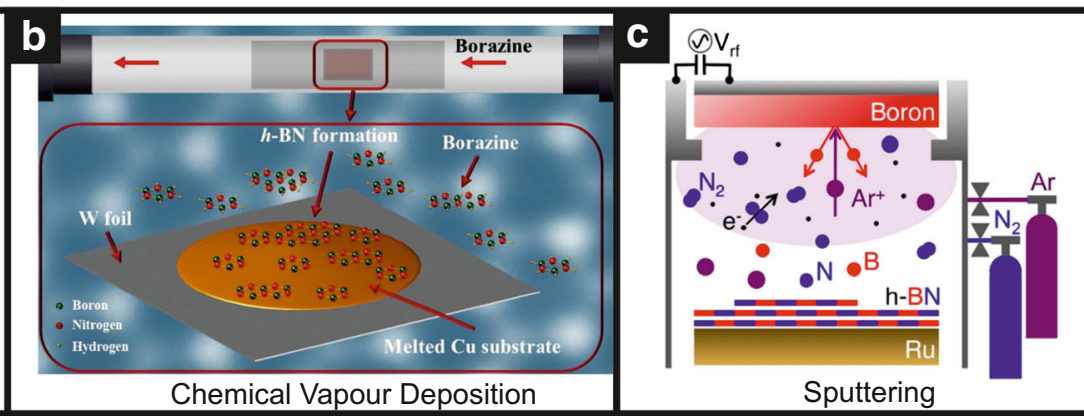

Fig. 3 Schematic representation of boron nitride manufacturing methods: exfoliation (a), chemical vapour deposition (b) and sputtering (c). Reprinted from $[37,41,42]$ with permission from the American Chemical Society and Elsevier respectively 
Table 1 Overview of recent literature reports of $2 \mathrm{D}-\mathrm{hBN}$ utilised as the basis of electroanalytical sensing platforms towards various (electro)analytical targets

\begin{tabular}{|c|c|c|c|c|}
\hline Material & Analyte & Linear range & LOD & Reference \\
\hline $\mathrm{Au}-\mathrm{NPs} / 2 \mathrm{D}-\mathrm{hBN} / \mathrm{GCE}$ & $\mathrm{H}_{2} \mathrm{O}_{2}$ & $0.04-50 \mathrm{mM}$ & $8.3 \mu \mathrm{M}$ & {$[51]$} \\
\hline $\mathrm{Au}-\mathrm{NPs} / 2 \mathrm{D}-\mathrm{hBN} / \mathrm{GCE}$ & Luteolin & $10-400 \mathrm{pM}$ & $1.7 \mathrm{pM}$ & {$[52]$} \\
\hline $\begin{array}{l}\text { MIP/Au NPs/2D } \\
\text { h-BN/GCE }\end{array}$ & Diethylstilbestrol & $5 \mathrm{pM}-0.02 \mu \mathrm{M}$ & $0.1 \mathrm{pM}$ & {$[35]$} \\
\hline $\mathrm{Au}-\mathrm{NPs} / 2 \mathrm{D}-\mathrm{hBN} / \mathrm{FTO}$ & Myoglobin & $0.1-100 \mu \mathrm{g} \mathrm{mL}^{-1}$ & $34.6 \mathrm{ng} \mathrm{mL}^{-1}$ & {$[53]$} \\
\hline $\begin{array}{l}\text { MIP/Fe@Au NPs/2D } \\
\text { hBN/GCE }\end{array}$ & Cypermethrin & $10^{-13}-10^{-8} \mathrm{M}$ & $0.03 \mathrm{pM}$ & {$[54]$} \\
\hline $\begin{array}{l}\text { HMICI-Pt-NPs/POM/2d } \\
\text { h-BN/CPE }\end{array}$ & N-Hydroxysuccinimide & $0.1-300 \mu \mathrm{M}$ & $60 \mathrm{nM}$ & {$[55]$} \\
\hline MIP/GQDS/2D-h-BN/GCE & Serotonin & $0.001-10 \mathrm{nM}$ & $0.1 \mathrm{pM}$ & {$[56]$} \\
\hline Trz-BN/GCE & L-Cysteine & - & - & {$[57]$} \\
\hline Cu-h-BNNS/GCE & Nitrite & $0.09-9853.45 \mu \mathrm{M}$ & $0.03 \mu \mathrm{M}$ & {$[58]$} \\
\hline 2D-hBN-QDs & Vitamin C & $0.8-5 \mathrm{mM}$ & $0.45 \mu \mathrm{M}$ & {$[59]$} \\
\hline D-2D-hBN/GCE & 4-AP and $\mathrm{Ph}$ & $\begin{array}{c}0.01-30 \mu \mathrm{M}(4-\mathrm{AP}) \\
0.1-30 \mu \mathrm{M}(\mathrm{Ph})\end{array}$ & $\begin{array}{c}0.003(4-\mathrm{AP}) \\
0.035(\mathrm{Ph}) \\
\mu \mathrm{M}\end{array}$ & {$[60]$} \\
\hline 2D h-BN/SPE & Dopamine & - & $0.65 \mu \mathrm{M}$ & {$[30]$} \\
\hline 2D h-BN whiskers/Ti & Nitrite & $10-400 \mu \mathrm{M}$ & $0.089 \mu \mathrm{M}$ & {$[61]$} \\
\hline flake 2D-hBN/GCE & Vitamin C & $30-1000 \mu \mathrm{M}$ & $3.77 \mu \mathrm{M}$ & {$[62]$} \\
\hline flake 2D-hBN/GCE & Dopamine & $0.5-150 \mu \mathrm{M}$ & $0.02 \mu \mathrm{M}$ & {$[62]$} \\
\hline flake 2D-hBN/GCE & Uric acid & $1-300 \mu \mathrm{M}$ & $0.15 \mu \mathrm{M}$ & {$[62]$} \\
\hline CN-hBN/GCE & Methyl parathion & $0.0002-2 \mathrm{nM}$ & $0.001 \mathrm{nM}$ & {$[63]$} \\
\hline 2D-hBN/f-MWCNTs/GCE & $\beta$-Agonists & $0.001-10 \mathrm{nM}$ & $0.0001 \mathrm{nM}$ & {$[64]$} \\
\hline BN/graphene/GCE & Nicotine & $1-1000 \mu \mathrm{M}$ & $0.42 \mu \mathrm{M}$ & {$[65]$} \\
\hline $\mathrm{Bi}_{2} \mathrm{O}_{3} / \mathrm{h}-\mathrm{BN} / \mathrm{SPCE}$ & Flutamide & $0.48-87 \mu \mathrm{M}$ & $0.009 \mu \mathrm{M}$ & {$[66]$} \\
\hline
\end{tabular}

$A u$, gold; NPs, nanoparticles; GCE, glassy carbon electrode; $M I P$, molecular imprinted polymer; Fe, iron; FTO; fluorine-doped tin oxide electrode; HMICI, 1-hexyl-3-methylimidazolium chloride; POM, polyoxometalate; $C P E$, carbon paste electrode; $G Q D S$, graphene quantum dots; $T r z$, triazine; $C u$, copper; $Q D s$, quantum dots; $T i$, titanium; 4-AP, 4-aminophenol; $P h$, phenol; $B N N S$, boron nitride nanosheets; $S P E$, screen-printed electrodes; $C N$, graphitic carbon nitride underlying carbon electrodes. Using optimised condition, suitable peak resolution between dopamine and uric acid was found to be possible with competitive electroanalytical outputs. This manuscript was the first to report that the novel nanomaterial $2 \mathrm{D}-\mathrm{hBN}$ is a beneficial electrocatalytic material for what might be considered an initially unlikely candidate. Such work has been extended by Li et al. [62] for the simultaneous sensing of vitamin $\mathrm{C}$, dopamine and uric acid using flake 2D-hBN upon glassy carbon electrodes (GCE) which has a high density of defects and active surface groups resulting in wide linear ranges and low limits of detection and also exhibited anti-interference abilities.

Other reports of utilising 2D-hBN have been in electrode configurations with other nanomaterials in the form of nanocomposites. For example, Yola and Atar [56] utilised graphene quantum dots with 2D-hBN with molecularly imprinted polymers upon a GCE for the sensing of serotonin. This approach was shown to be (electro)analytically competitive over other literature reports with the authors attributing the enhanced outputs of their sensor due to charge transfer facilitated by the graphene quantum
dots/2Dh-hBN, reduced mass transfer resistance on the underlying supporting electrode (GCE) and synergistic effect between the 2D-hBN and the graphene quantum dots [56]. Further inspection of their reported voltammetric responses [56], where a bare GCE is compared to a GCE electrode modified with 2D-hBN and graphene QDs/2D-hBN towards a simple redox probe, indicates a significantly differing voltammetry, which can be inferred as a change from diffusional (at the GCE) to thin-layer/adsorption type effects (at the 2D-hBN and graphene QDs/2D-hBN modified electrodes. The later gives rise to larger peak current/ analytical response, which is expected [69]. This potentially contributed to the factors that the authors attributed to the improved electrochemical response, but further analysis would have been useful. The authors successfully utilised their nanocomposite sensor for the reliable measurement of serotonin in urine samples [56]. This avenue of using 2D-hBN in nanocomposites has been extended to the sensing of methyl parathion using graphitic carbon nitride, 2D-hBN and molecularly imprinted polymer [63]. This approach was shown to exhibit electroanalytical performances superior to previous literature reports and was shown 
to be successfully applied to orange juice samples for methyl parathion detection. In a similar vein, $\beta$-agonists have been shown to be electroanalytically detected in urine samples using 2D-hBN/multi-walled carbon nanotube nanocomposite modified GCE in the presence of ascorbic acid and uric acid [64]. Other approaches have combined 2D-hBN with graphene for the detection of nicotine applied in real tobacco samples [65] and 2D$\mathrm{hBN}$ with bismuth oxide for the sensing of flutamine applied in environmental samples [66]. In all cases, the synergy of mixing the various components comprising the nanocomposite is attributed to the beneficial electrochemical response. Generally, authors fail to show data of the various ratios of the components comprising the nanocomposite upon the electrochemical/ electroanalytical response demonstrating how they chose their final composition.

Shen et al. have utilised defect-enhanced h-BN (termed (D-h-BN) on a GCE as a sensing platform for the detection of 4-aminophenol (4-AP) and phenol (Ph) [60] and lead [70]. They report the synthesis of defective h-BN via a single precursor calcination process. As shown in Fig. 4, defective h-BN exhibits the presence of pore holes within the basal plane of the typical laminar structure of 2DhBN. These defects/holes are reported to be chemically active and provide electrochemical active sites via defect-related sub-levels in the band gap [60]. In both cases, the improved electrochemical response was compared to a bare GCE, which demonstrated the D-h$\mathrm{BN}$ to give to superior electroanalytical signatures, attributed by the authors to be due to the material exhibiting fast electron transfer, large electrochemical active surface area and abundant electroactive sites, which were induced by the defective nature of the D-h-BN structure. The authors demonstrated the successful determination of 4-AP and $\mathrm{Ph}$ in tap and lake water samples.

Luo et al. [61] reported the useful fabrication of hexagonal boron nitride (h-BN) whiskers. Their hBN whiskers were synthesised via a polymeric precursor methodology utilising boric acid $\left(\mathrm{H}_{3} \mathrm{BO}_{3}\right)$ and melamine $\left(\mathrm{C}_{3} \mathrm{H}_{6} \mathrm{~N}_{6}\right)$ as raw materials with the precursors slowly heated in a tube furnace to high temperatures (1073-1273 K) in a flowing nitrogen/hydrogen (5\% hydrogen) atmosphere. The fabricated h-BN whiskers are shown in Fig. 5, which are $0.5-3 \mu \mathrm{m}$ in diameter and 200 $500 \mathrm{~mm}$ in length. The authors sought to demonstrate the usefulness of their h-BN whiskers by exploring the electroanalytical sensing of nitrite. Figure 5 shows cyclic voltammetric curves of h-BN whiskers (poorly and highly crystallised) and compared to those of a bare Ti electrode. The choice of electrode gives the impression that the h-BN whiskers give rise to outstanding electrochemical signatures, even electrocatalytic, one might suggest. That said, nitrite can be readily electrochemically oxidised using a range of carbon electrodes [71-75]. It would have been insightful to compare the
Fig. 4 TEM image of defectenhanced h-BN (d-h-BN) (a), simultaneous calibration plot (DPV) of 4-AP and Ph using d-h$\mathrm{BN} / \mathrm{GCE}$ (b) and electrochemical impedance spectra of d-h-BN/ GCE (c). Reprinted from [60] with permission from Elsevier

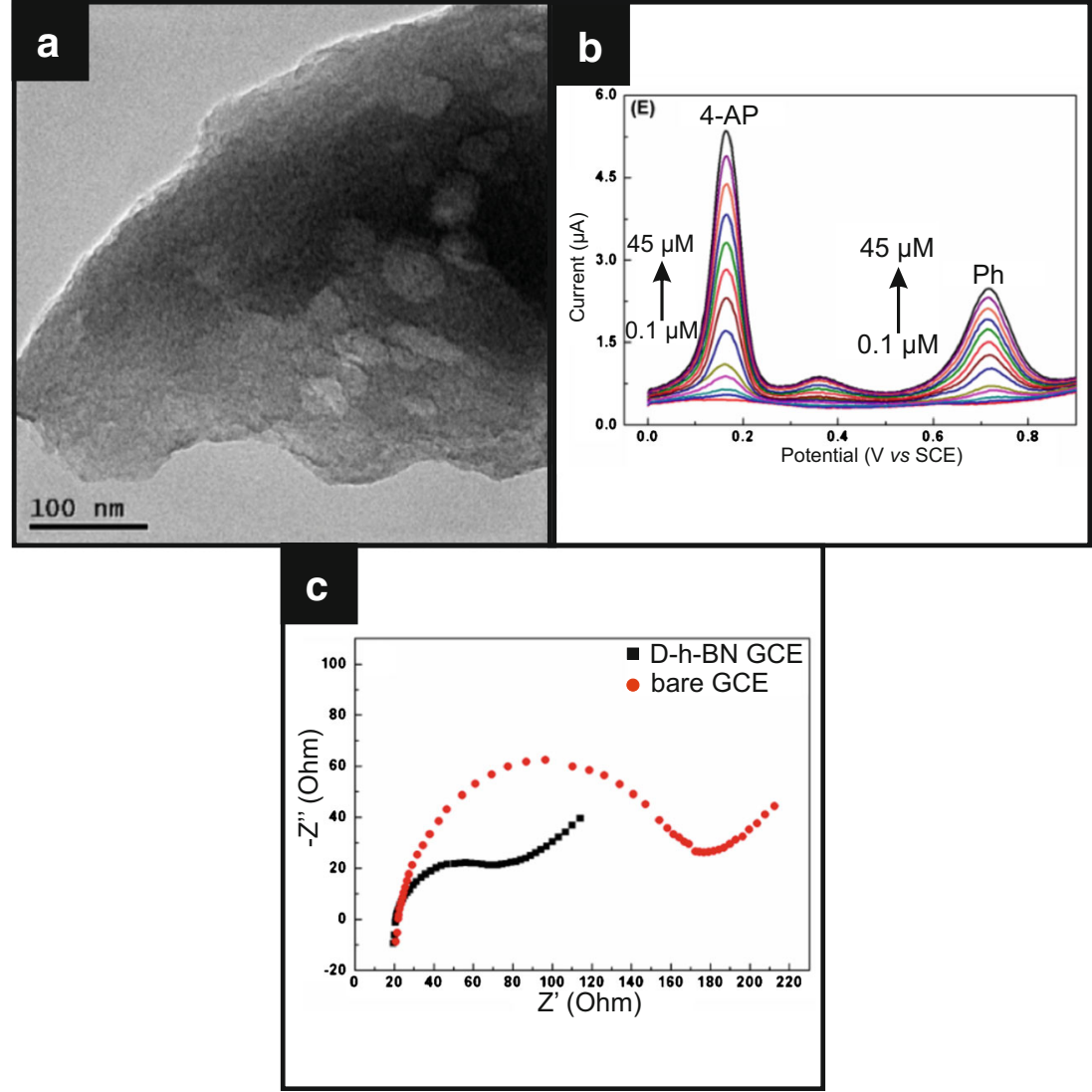



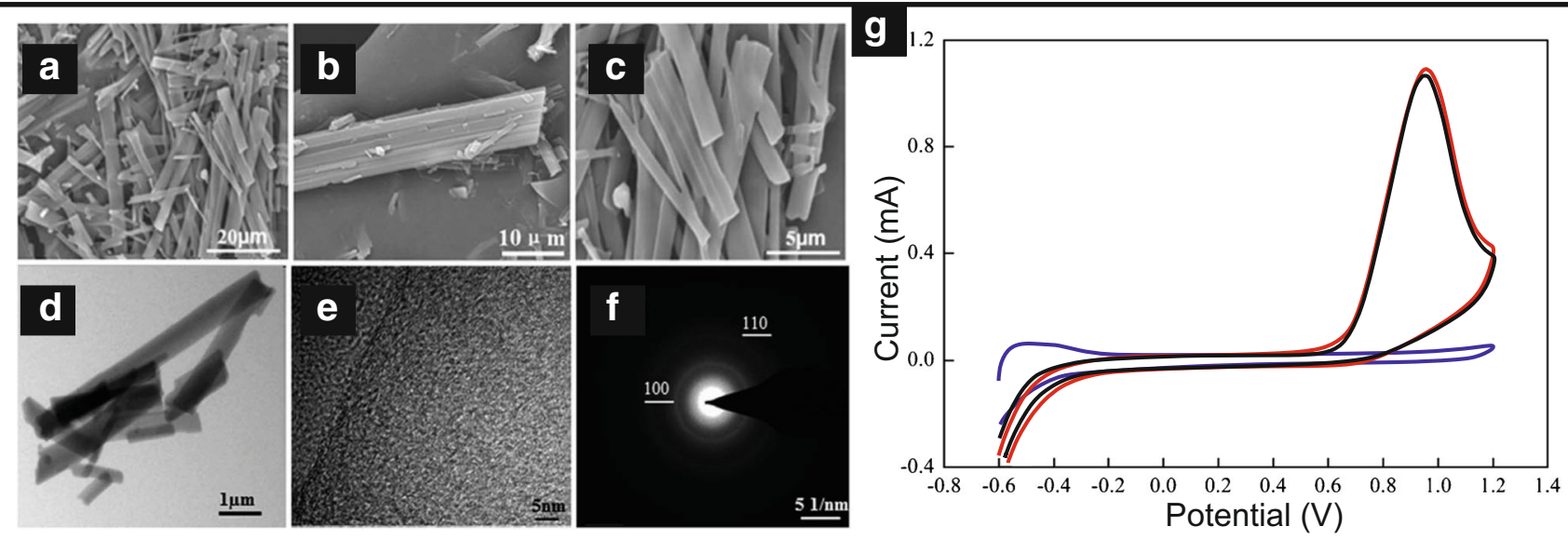

Fig. 5 SEM and TEM micrographs of the h-BN whiskers. a Lowmagnification SEM images; $\mathbf{b}$, $\mathbf{c}$ high-magnification SEM images. $\mathbf{d}$ TEM images. e HRTEM. f The SAED pattern. Cyclic voltammetric curves (g) of the poorly crystallised BN whiskers electrode (red line),

electrochemical response of the h-BN whiskers to other carbon-based electrodes under the same employed experimental parameters to demonstrate that they exhibit a large electrochemically area, as stated by the authors [61]. Additionally, it would have been insightful to explore different coverages to try and optimise the electroanalytical outputs; that said, the sensor was shown to allow the determination of nitrite at various concentrations within tap water [61].

From the above analysis and inspection of Table 1, it is clear that $2 \mathrm{D}-\mathrm{hBN}$ is being beneficially used in the field of electroanalysis. A critical question from the above literature is, however, why does this initially overlooked material, reported the highly crystallised BN whiskers electrode (black line) and bare Ti electrode (blue) towards the electrochemical oxidation of nitrite $\left(1.0 \mathrm{~m} \mathrm{~mol} \mathrm{~L}-1\right.$ nitrite in $0.1 \mathrm{~mol} \mathrm{~L}^{-1}$ phosphate buffer). Reproduced from reference [61] with permission of the Royal Society of Chemistry

to be an insulator, clearly functions as an electrochemical/ electroanalytical sensing platform? As the literature has progressed, many infer their beneficial responses to be due to a range of factors, such as high surface area, increased electron transfer, high accessible active sites and fast electron transfer due to defects [43, 67, 76-91]. Many different surface morphologies have been reported, but only the work reported by Garcia-Miranda et al. [91] has shown the origin of electrocatalytic properties at true mono-layer 2D-hBN (CVD grown on $\mathrm{Si} / \mathrm{SiO}_{2}$ ). As shown in Fig. 6, they compared the electrochemical signatures of monolayer $2 \mathrm{D}-\mathrm{hBN}$ with that of $2 \mathrm{D}$ $\mathrm{hBN}$ with physical linear defects (PLDs). In the former case, a

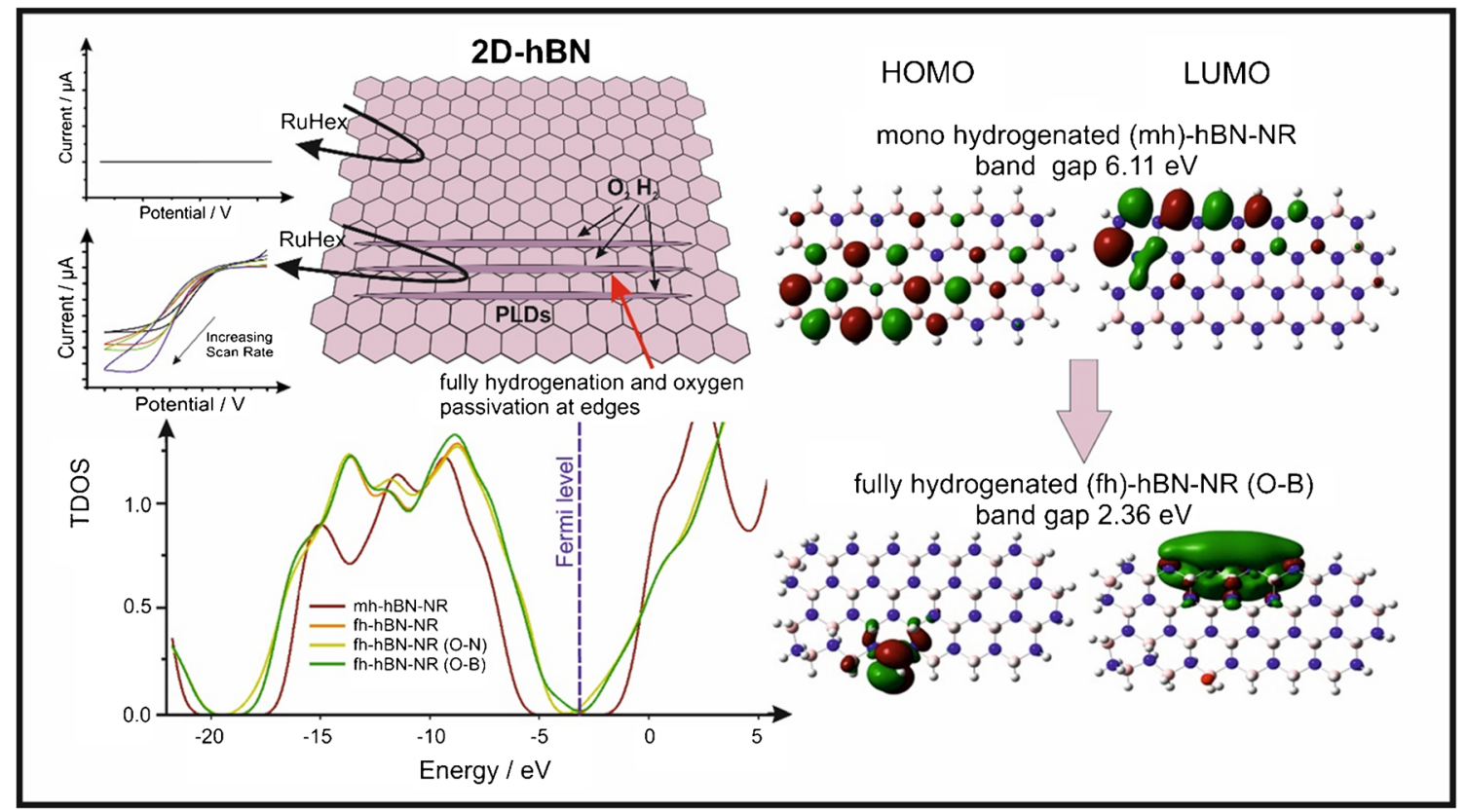

Fig. 6 Graphical representation of physical linear defects (PLDs) upon 2D-hBN, giving rise to electrochemically useful signatures supported with DFT theory. Adapted with permission by the Royal Society of Chemistry from reference [91] 
pristine (no defects) 2D-hBN monolayer is utilised which gives rise to zero/negligible electrochemical outputs. This has probably been encountered previously and might have led to abandoning the use of this material in electrochemistry which, on first sight, agrees with literature reports of 2D-hBN being non-conductive. In the latter case, PLDs are introduced which transforms this previously electrochemically inert material into giving useful electrochemically signatures; these induced defects upon the basal plane of the 2D h-BN surface are the only source of the newly electroactive material and the reason of the change from insulator to semi-conductor. DFT calculations were used to calculate the band gap of the introduced PLDs, which reported that the fully hydrogenation and oxygen passivation of the created edges are capable of reducing the band gap from 6.11 to $2.36 \mathrm{eV}$. This insight explains why 2D-hBN utilised in the above studies (see Table 1) give rise to electrochemical/electroanalytical outputs, since a range of 2D-hBN materials are not true monolayer and have abundant edges and defects across their basal plane for electron transfer to occur; this will be further enhanced for multilayers of $2 \mathrm{D}-\mathrm{hBN}$.

\section{Conclusions}

In this review, we have demonstrated that the previously overlooked 2D-hBN is beneficially being utilised as the basis of electroanalytical sensing platforms. Tailored defect-rich boron nitride microstructures have great potential as active materials for electroanalytical sensor fabrication, rather than their conventional use as electrode substrates (i.e. monolayer 2D$\mathrm{hBN}$ ). Although the electrocatalytic behaviour of $2 \mathrm{D}-\mathrm{hBN}$ is still relatively unexplored territory, with careful execution of defect incorporation into the boron nitride nanosheets, researchers are unveiling its novel sensing applications towards a variety of analytes. Based upon current literature reports, we provide a summary of areas that could potentially be explored/ reported in future work utilising $2 \mathrm{D}-\mathrm{hBN}$ and related structures: (1) exploring the lateral sizes, $\mathrm{La}$ and $\mathrm{Lc}$ and defects (at edges and across basal surfaces) and understanding how these parameters affect the electrochemical/electroanalytical responses and can be be tailored and utilised to optimise electrochemical outputs; (2) exploring the role of the underlying/ supporting electrode; (3) undertaking and reporting coverage studies, such that optimisation of the electrochemical/ electroanalytical responses can be achieved and also noting that thin-layer responses can be observed that might be mistaken for "electrocatalysis"; (4) exploring the synergy of various materials mixed together to form nanocompositeswhich are the origin/dominated the electrochemical response and understanding how the various ratios of each material comprising the nanocomposite can give rise to optimal electroanalytical outputs. As research grows in the area of 2D-
$\mathrm{hBN}$ electrochemistry, more parameters will likely need to be explored/unravelled but it is clear that this is an emerging and exciting field utilising an interesting and previously overlooked 2D nanomaterial.

Funding This work received funding from the Engineering and Physical Sciences Research Council (References: EP/N001877/1 and EP/ P007767/1), British Council Institutional Grant Link (No. 172726574) and Innovate UK (KTP Reference: 11606).

\section{Compliance with ethical standards}

Conflict of interest The authors declare that there is no conflict of interest.

Open Access This article is licensed under a Creative Commons Attribution 4.0 International License, which permits use, sharing, adaptation, distribution and reproduction in any medium or format, as long as you give appropriate credit to the original author(s) and the source, provide a link to the Creative Commons licence, and indicate if changes were made. The images or other third party material in this article are included in the article's Creative Commons licence, unless indicated otherwise in a credit line to the material. If material is not included in the article's Creative Commons licence and your intended use is not permitted by statutory regulation or exceeds the permitted use, you will need to obtain permission directly from the copyright holder. To view a copy of this licence, visit http://creativecommons.org/licenses/by/4.0/.

\section{References}

1. Novoselov KS, Geim AK, Morozov SV, Jiang D, Zhang Y, Dubonos SV, et al. Electric field effect in atomically thin carbon films. Science. 2004;306(5696):666-9. https://doi.org/10.1126/ science. 1102896.

2. Zhang X, Gao J, Xiao Y, Wang J, Sun G, Zhao Y, et al. Regulation of 2D graphene materials for electrocatalysis. Chem Asian J. 2020;15(15):2271-81. https://doi.org/10.1002/asia.202000249.

3. Mohamad Nasir MZ, Pumera M. Emerging mono-elemental 2D nanomaterials for electrochemical sensing applications: from borophene to bismuthene. Trends Anal Chem. 2019;121:115696. https://doi.org/10.1016/j.trac.2019.115696.

4. Li D, Gao J, Cheng P, He J, Yin Y, Hu Y, et al. 2D boron sheets: structure, growth, and electronic and thermal transport properties. Adv Funct Mater. 2020;30(8):1904349. https://doi.org/10.1002/ adfm. 201904349.

5. Glavin NR, Rao R, Varshney V, Bianco E, Apte A, Roy A, et al. Emerging applications of elemental 2D materials. Adv Mater. 2020;32(7):1904302. https://doi.org/10.1002/adma.201904302.

6. Chia X, Pumera M. Layered transition metal dichalcogenide electrochemistry: journey across the periodic table. Chem Soc Rev. 2018;47(15):5602-13. https://doi.org/10.1039/C7CS00846E.

7. Ares P, Palacios JJ, Abellán G, Gómez-Herrero J, Zamora F. Recent progress on antimonene: a new bidimensional material. Adv Mater. 2018;30(2):1703771. https://doi.org/10.1002/adma. 201703771.

8. Geim AK, Grigorieva IV. Van der Waals heterostructures. Nature. 2013;499(7459):419-25. https://doi.org/10.1038/nature12385.

9. Rowley-Neale SJ, Ratova M, Fugita LTN, Smith GC, Gaffar A, Kulczyk-Malecka J, et al. Magnetron sputter-coated nanoparticle MoS2 supported on nanocarbon: a highly efficient electrocatalyst 
toward the hydrogen evolution reaction. ACS Omega. 2018;3(7): 7235-42. https://doi.org/10.1021/acsomega.8b00258.

10. Ambrosi A, Sofer Z, Pumera M. Electrochemical exfoliation of layered black phosphorus into phosphorene. Angew Chem Int Ed. 2017;129(35):10579-81. https://doi.org/10.1002/ange.201705071.

11. Martínez-Periñán E, Down MP, Gibaja C, Lorenzo E, Zamora F, Banks CE. Antimonene: a novel 2D nanomaterial for supercapacitor applications. Adv Energy Mater. 2018;8(11): 1702606. https://doi.org/10.1002/aenm.201702606.

12. Pemberton R, Amine A, Hart J. Voltammetric behavior of chlorophyll a at a screen-printed carbon electrode and its potential role as a biomarker for monitoring fecal contamination. Anal Lett. 2007;37: 1625-43. https://doi.org/10.1081/AL-120037592.

13. García-Miranda Ferrari A, Carrington P, Rowley-Neale SJ, Banks CE. Recent advances in portable heavy metal electrochemical sensing platforms. Environ Sci: Water Res Technol. 2020;6(10):267690. https://doi.org/10.1039/D0EW00407C.

14. Kadara RO, Jenkinson N, Banks CE. Disposable bismuth oxide screen printed electrodes for the high throughput screening of heavy metals. Electroanalysis. 2009;21(22):2410-4. https://doi.org/10. 1002/elan.200900266.

15. Foster CW, de Souza AP, Metters JP, Bertotti M, Banks CE. Metallic modified (bismuth, antimony, tin and combinations thereof) film carbon electrodes. Analyst. 2015;140(22):7598-612. https://doi.org/10.1039/C5AN01692D.

16. Elbardisy HM, Ferrari AGM, Foster CW, Sutcliffe OB, Brownson DAC, Belal TS, et al. Forensic electrochemistry: the electroanalytical sensing of mephedrone metabolites. ACS Omega. 2019;4(1): 1947-54. https://doi.org/10.1021/acsomega.8b02586.

17. Smith JP, Randviir EP, Banks CE. An introduction to forensic electrochemistry. Forensic Sci. 2016:89-102. https://doi.org/10. 1002/9783527693535.ch5.

18. Zuway KY, Smith JP, Foster CW, Kapur N, Banks CE, Sutcliffe OB. Detection and quantification of new psychoactive substances (NPSs) within the evolved "legal high" product, NRG-2, using high performance liquid chromatography-amperometric detection (HPLC-AD). Analyst. 2015;140(18):6283-94. https://doi.org/10. 1039/C5AN01106J.

19. Randviir EP, Metters JP, Stainton J, Banks CE. Electrochemical impedance spectroscopy versus cyclic voltammetry for the electroanalytical sensing of capsaicin utilising screen printed carbon nanotube electrodes. Analyst. 2013;138(10):2970-81. https://doi.org/ $10.1039 / \mathrm{c} 3 \mathrm{an} 00368 \mathrm{j}$.

20. Ngamchuea K, Hurst P, Batchelor-McAuley C, Compton RG. Handheld electrochemical device for the determination of the strength of garlic. Sensors Actuators B Chem. 2016;232:138-42. https://doi.org/10.1016/j.snb.2016.03.057.

21. Pierini GD, Maccio SA, Robledo SN, Ferrari AG-M, Banks CE, Fernández H, et al. Screen-printed electrochemical-based sensor for taxifolin determination in edible peanut oils. Microchem J. 2020;159:105442. https://doi.org/10.1016/j.microc.2020.105442.

22. Hernández-Ibáñez N, García-Cruz L, Montiel V, Foster CW, Banks $\mathrm{CE}$, Iniesta J. Electrochemical lactate biosensor based upon chitosan/carbon nanotubes modified screen-printed graphite electrodes for the determination of lactate in embryonic cell cultures. Biosens Bioelectron. 2016;77:1168-74. https://doi.org/10.1016/j. bios.2015.11.005.

23. Hernández-Ibáñez N, Sanjuán I, Montiel MÁ, Foster CW, Banks CE, Iniesta J. 1-Cysteine determination in embryo cell culture media using Co (II)-phthalocyanine modified disposable screen-printed electrodes. J Electroanal Chem. 2016;780:303-10. https://doi.org/ 10.1016/j.jelechem.2016.09.028.

24. Gould T, Lebègue S, Björkman T, Dobson JF. 2D materials, vol 95. Semicond. Semimet. Elsevier Science; 2016. https://doi.org/10. 1016/bs.semsem.2016.04.001
25. Tyagi D, Wang H, Huang W, Hu L, Tang Y, Guo Z, et al. Recent advances in two-dimensional-material-based sensing technology toward health and environmental monitoring applications. Nanoscale. 2020;12(6):3535-59. https://doi.org/10.1039/ C9NR10178K.

26. Rao CNR, Sood AK, Subrahmanyam KS, Govindaraj A. Graphene: the new two-dimensional nanomaterial. Angew Chem Int Ed. 2009;48(42):7752-77. https://doi.org/10.1002/anie. 200901678

27. Lin Y, Connell JW. Advances in 2D boron nitride nanostructures: nanosheets, nanoribbons, nanomeshes, and hybrids with graphene. Nanoscale. 2012;4(22):6908-39. https://doi.org/10.1039/ C2NR32201C.

28. Stagi L, Ren J, Innocenzi P. From 2-D to 0-D boron nitride materials, the next challenge. Materials. 2019;12(23):3905.

29. Pakdel A, Bando Y, Golberg D. Nano boron nitride flatland. Chem Soc Rev. 2014;43(3):934-59. https://doi.org/10.1039/ C3CS60260E

30. Khan AF, Brownson DAC, Randviir EP, Smith GC, Banks CE. 2D hexagonal boron nitride (2D-hBN) explored for the electrochemical sensing of dopamine. Anal Chem. 2016;88(19):9729-37. https:// doi.org/10.1021/acs.analchem.6b02638.

31. Ambrosi A, Chua CK, Latiff NM, Loo AH, Wong CHA, Eng AYS, et al. Graphene and its electrochemistry - an update. Chem Soc Rev. 2016;45(9):2458-93. https://doi.org/10.1039/C6CS00136J.

32. Ambrosi A, Pumera M. The CVD graphene transfer procedure introduces metallic impurities which alter the graphene electrochemical properties. Nanoscale. 2014;6(1):472-6. https://doi.org/ 10.1039/C3NR05230C.

33. García-Miranda Ferrari A, Foster CW, Brownson DAC, Whitehead KA, Banks CE. Exploring the reactivity of distinct electron transfer sites at CVD grown monolayer graphene through the selective electrodeposition of MoO2 nanowires. Sci Rep. 2019;9(1):12814. https://doi.org/10.1038/s41598-019-48022-6.

34. Xu M, Liang T, Shi M, Chen H. Graphene-like two-dimensional materials. Chem Rev. 2013;113(5):3766-98. https://doi.org/10. 1021/cr300263a.

35. Angizi S, Khalaj M, Alem SAA, Pakdel A, Willander M, Hatamie A, et al. Review - towards the two-dimensional hexagonal boron nitride (2D h-BN) electrochemical sensing platforms. J Electrochem Soc. 2020;167(12):126513. https://doi.org/10.1149/ 1945-7111/abaf29.

36. Wang H, Zhao Y, Xie Y, Ma X, Zhang X. Recent progress in synthesis of two-dimensional hexagonal boron nitride. J Semicond. 2017;38(3):031003. https://doi.org/10.1088/16744926/38/3/031003.

37. Sutter P, Lahiri J, Zahl P, Wang B, Sutter E. Scalable synthesis of uniform few-layer hexagonal boron nitride dielectric films. Nano Lett. 2013;13(1):276-81. https://doi.org/10.1021/nl304080y.

38. Wang H, Zhang X, Meng J, Yin Z, Liu X, Zhao Y, et al. Controlled growth of few-layer hexagonal boron nitride on copper foils using ion beam sputtering deposition. Small. 2015;11(13):1542-7. https://doi.org/10.1002/smll.201402468.

39. Kim G, Jang AR, Jeong HY, Lee Z, Kang DJ, Shin HS. Growth of high-crystalline, single-layer hexagonal boron nitride on recyclable platinum foil. Nano Lett. 2013;13(4):1834-9. https://doi.org/10. 1021/n1400559s.

40. Koepke JC, Wood JD, Chen Y, Schmucker SW, Liu X, Chang NN, et al. Role of pressure in the growth of hexagonal boron nitride thin films from ammonia-borane. Chem Mater. 2016;28(12):4169-79. https://doi.org/10.1021/acs.chemmater.6b00396.

41. Morishita T, Okamoto H, Katagiri Y, Matsushita M, Fukumori K. A high-yield ionic liquid-promoted synthesis of boron nitride nanosheets by direct exfoliation. Chem Commun. 2015;51(60):1206871. https://doi.org/10.1039/C5CC04077A. 
42. Khan MH, Liu HK, Sun X, Yamauchi Y, Bando Y, Golberg D, et al. Few-atomic-layered hexagonal boron nitride: CVD growth, characterization, and applications. Mater Today. 2017;20(10):61128. https://doi.org/10.1016/j.mattod.2017.04.027.

43. Khan AF, Down MP, Smith GC, Foster CW, Banks CE. Surfactantexfoliated 2D hexagonal boron nitride (2D-hBN): role of surfactant upon the electrochemical reduction of oxygen and capacitance applications. J Mater Chem A. 2017;5(8):4103-13. https://doi.org/10. 1039/C6TA09999H.

44. Sachs B, Wehling TO, Katsnelson MI, Lichtenstein AI. Midgap states and band gap modification in defective graphene/h-BN heterostructures. Phys Rev B. 2016;94(22):224105.

45. Kubota Y, Watanabe K, Tsuda O, Taniguchi T. Deep ultraviolet light-emitting hexagonal boron nitride synthesized at atmospheric pressure. Science. 2007;317(5840):932-4. https://doi.org/10.1126/ science.1144216.

46. Yang Y, Hou H, Zou G, Shi W, Shuai H, Li J, et al. Electrochemical exfoliation of graphene-like two-dimensional nanomaterials. Nanoscale. 2019;11(1):16-33. https://doi.org/10.1039/ C8NR08227H

47. Chen W, Li Y, Yu G, Li C-Z, Zhang SB, Zhou Z, et al. Hydrogenation: a simple approach to realize semiconductor-halfmetal-metal transition in boron nitride nanoribbons. J Am Chem Soc. 2010;132(5):1699-705. https://doi.org/10.1021/ja908475v.

48. Koitz R, Norskov JK, Studt F. A systematic study of metalsupported boron nitride materials for the oxygen reduction reaction. Phys Chem Chem Phys. 2015;17(19):12722-7. https://doi.org/10. 1039/C5CP01384D.

49. Uosaki K, Elumalai G, Noguchi H, Masuda T, Lyalin A, Nakayama A, et al. Boron nitride nanosheet on gold as an electrocatalyst for oxygen reduction reaction: theoretical suggestion and experimental proof. J Am Chem Soc. 2014;136(18):6542-5. https://doi.org/10. 1021/ja500393g.

50. Wang Y, Mayorga-Martinez CC, Chia X, Sofer Z, Pumera M. Nonconductive layered hexagonal boron nitride exfoliation by bipolar electrochemistry. Nanoscale. 2018;10(15):7298-303. https:// doi.org/10.1039/C8NR00082D.

51. Yang G-H, Abulizi A, Zhu J-J. Sonochemical fabrication of gold nanoparticles-boron nitride sheets nanocomposites for enzymeless hydrogen peroxide detection. Ultrason Sonochem. 2014;21(6): 1958-63. https://doi.org/10.1016/j.ultsonch.2014.01.020.

52. Fu L, Liu Z, Huang Y, Lai G, Zhang H, Su W, et al. Square wave voltammetric quantitative determination of flavonoid luteolin in peanut hulls and Perilla based on Au NPs loaded boron nitride nanosheets. J Electroanal Chem. 2018;817:128-33. https://doi. org/10.1016/j.jelechem.2018.04.009.

53. Adeel M, Rahman MM, Lee JJ. Label-free aptasensor for the detection of cardiac biomarker myoglobin based on gold nanoparticles decorated boron nitride nanosheets. Biosens Bioelectron. 2019;126:143-50. https://doi.org/10.1016/j.bios.2018.10.060.

54. Atar N, Yola ML. Core-shell nanoparticles/two-dimensional (2D) hexagonal boron nitride nanosheets with molecularly imprinted polymer for electrochemical sensing of cypermethrin. J Electrochem Soc. 2018;165(5):H255-62. https://doi.org/10.1149/ 2.1311805 jes.

55. Karimi-Maleh H, Karimi F, Malekmohammadi S, Zakariae N, Esmaeili R, Rostamnia S, et al. An amplified voltammetric sensor based on platinum nanoparticle/polyoxometalate/two-dimensional hexagonal boron nitride nanosheets composite and ionic liquid for determination of N-hydroxysuccinimide in water samples. J Mol Liq. 2020;310:113185. https://doi.org/10.1016/j.molliq.2020. 113185.

56. Yola ML, Atar N. A novel detection approach for serotonin by graphene quantum dots/two-dimensional (2D) hexagonal boron nitride nanosheets with molecularly imprinted polymer. Appl Surf
Sci. 2018;458:648-55. https://doi.org/10.1016/j.apsusc.2018.07. 142.

57. Daneshnia S, Adeli M, Yari A, Shams A, Donskyi IS, Unger WES. Low temperature functionalization of two-dimensional boron nitride for electrochemical sensing. Mater Res Express. 2019;6(9): 095076. https://doi.org/10.1088/2053-1591/ab317b.

58. Zhang Y. Copper/hexagonal boron nitride nanosheet composite as an electrochemical sensor for nitrite determination. Int $\mathrm{J}$ Electrochem Sci. 2018;13:5995-6004. https://doi.org/10.20964/ 2018.06.23.

59. Angizi S, Hatamie A, Ghanbari H, Simchi A. Mechanochemical green synthesis of exfoliated edge-functionalized boron nitride quantum dots: application to vitamin $\mathrm{C}$ sensing through hybridization with gold electrodes. ACS Appl Mater Interfaces. 2018;10(34): 28819-27. https://doi.org/10.1021/acsami.8b07332.

60. Shen Y, Yan H, Guo H, Long Y, Li W. Defect-rich hexagonal boron nitride for the simultaneous determination of 4 aminophenol and phenol. Sensors Actuators B Chem. 2020;303: 127248. https://doi.org/10.1016/j.snb.2019.127248.

61. Luo W, Yang T, Su L, Chou K-C, Hou X. Preparation of hexagonal $\mathrm{BN}$ whiskers synthesized at low temperature and their application in fabricating an electrochemical nitrite sensor. RSC Adv. 2016;6(33):27767-74. https://doi.org/10.1039/C5RA27234C.

62. Li Q, Huo C, Yi K, Zhou L, Su L, Hou X. Preparation of flake hexagonal $\mathrm{BN}$ and its application in electrochemical detection of ascorbic acid, dopamine and uric acid. Sensors Actuators B Chem. 2018;260:346-56. https://doi.org/10.1016/j.snb.2017.12.208.

63. Kiran TR, Atar N, Yola ML. A methyl parathion recognition method based on carbon nitride incorporated hexagonal boron nitride nanosheets composite including molecularly imprinted polymer. J Electrochem Soc. 2019;166(12):H495-501. https://doi.org/10. 1149/2.0331912jes.

64. Yola ML, Atar N. Simultaneous determination of $\beta$-agonists on hexagonal boron nitride nanosheets/multi-walled carbon nanotubes nanocomposite modified glassy carbon electrode. Mater Sci Eng C. 2019;96:669-76. https://doi.org/10.1016/j.msec.2018.12.004.

65. Jerome R, Sundramoorthy AK. Preparation of hexagonal boron nitride doped graphene film modified sensor for selective electrochemical detection of nicotine in tobacco sample. Anal Chim Acta. 2020;1132:110-20. https://doi.org/10.1016/j.aca.2020.07.060.

66. Kokulnathan T, Vishnuraj R, Wang T-J, Kumar EA, Pullithadathil B. Heterostructured bismuth oxide/hexagonal-boron nitride nanocomposite: a disposable electrochemical sensor for detection of flutamide. Ecotoxicol Environ Saf. 2021;207:111276. https://doi. org/10.1016/j.ecoenv.2020.111276.

67. Khan AF, Randviir EP, Brownson DAC, Ji X, Smith GC, Banks CE. 2D hexagonal boron nitride (2D-hBN) explored as a potential electrocatalyst for the oxygen reduction reaction. Electroanalysis. 2017;29(2):622-34. https://doi.org/10.1002/elan.201600462.

68. Patil Indrajit M, Jijil CP, Lokanathan M, Swami A, Kakade B. Mechanical activation in reduced graphite oxide/boron nitride nanocomposite electrocatalysts for significant improvement in dioxygen reduction. Sustain Energy Fuels. 2018;2(1):252-61. https://doi.org/10.1039/C7SE00461C.

69. Tanner EEL, Compton RG. How can electrode surface modification benefit electroanalysis? Electroanalysis. 2018;30(7):1336-41. https://doi.org/10.1002/elan.201700807.

70. Shen Y, Ouyang H, Li W, Long Y. Microchimica Acta, 2020; In Press.

71. Kozub BR, Rees NV, Compton RG. Electrochemical determination of nitrite at a bare glassy carbon electrode; why chemically modify electrodes? Sensors Actuators B Chem. 2010;143(2):539-46. https://doi.org/10.1016/j.snb.2009.09.065.

72. Khairy M, Kadara RO, Banks CE. Electroanalytical sensing of nitrite at shallow recessed screen printed microelectrode arrays. 
Anal Methods. 2010;2(7):851-4. https://doi.org/10.1039/ C0AY00142B.

73. Metters JP, Randviir EP, Banks CE. Screen-printed back-to-back electroanalytical sensors. Analyst. 2014;139(21):5339-49. https:// doi.org/10.1039/C4AN01501K.

74. Tan F, Metters JP, Banks CE. Electroanalytical applications of screen printed microelectrode arrays. Sensors Actuators B Chem. 2013;181:454-62. https://doi.org/10.1016/j.snb.2013.02.034.

75. Metters JP, Kadara RO, Banks CE. Electroanalytical properties of screen printed graphite microband electrodes. Sensors Actuators B Chem. 2012;169:136-43. https://doi.org/10.1016/j.snb.2012.04. 045.

76. Felix IM, Pereira LFC. Thermal conductivity of graphene-hBN superlattice ribbons. Sci Rep. 2018;8(1):2737. https://doi.org/10. 1038/s41598-018-20997-8.

77. Golla D, Brasington A, LeRoy BJ, Sandhu A. Ultrafast relaxation of hot phonons in graphene-hBN heterostructures. APL Mater. 2017;5(5):056101. https://doi.org/10.1063/1.4982738.

78. Kumar A, Low T, Fung KH, Avouris P, Fang NX. Tunable lightmatter interaction and the role of hyperbolicity in graphene- $\mathrm{hBN}$ system. Nano Lett. 2015;15(5):3172-80. https://doi.org/10.1021/ acs.nanolett.5b01191.

79. Levendorf MP, Kim C-J, Brown L, Huang PY, Havener RW, Muller DA, et al. Graphene and boron nitride lateral heterostructures for atomically thin circuitry. Nature. 2012;488: 627. https://doi.org/10.1038/nature11408 https://www.nature.com/ articles/nature11408\#supplementary-information. Accessed 16 Oct 2020.

80. Liu M, Li Y, Chen P, Sun J, Ma D, Li Q, et al. Quasi-freestanding monolayer heterostructure of graphene and hexagonal boron nitride on $\operatorname{Ir}(111)$ with a zigzag boundary. Nano Lett. 2014;14(11):63427. https://doi.org/10.1021/n1502780u.

81. Ci L, Song L, Jin C, Jariwala D, Wu D, Li Y, et al. Atomic layers of hybridized boron nitride and graphene domains. Nat Mater. 2010;9: 430. https://doi.org/10.1038/nmat2711 https://www.nature.com/ articles/nmat2711\#supplementary-information. Accessed 16 Oct 2020.

82. Liu Z, Ma L, Shi G, Zhou W, Gong Y, Lei S, et al. In-plane heterostructures of graphene and hexagonal boron nitride with controlled domain sizes. Nat Nanotechnol. 2013;8:119. https://doi.org/ 10.1038/nnano.2012.256 https://www.nature.com/articles/nnano. 2012.256\#supplementary-information. Accessed 16 Oct 2020.

83. Dean CR, Young AF, Meric I, Lee C, Wang L, Sorgenfrei S, et al. Boron nitride substrates for high-quality graphene electronics. Nat Nanotechnol. 2010;5:722. https://doi.org/10.1038/nnano.2010.172 https://www.nature.com/articles/nnano.2010.172\#supplementaryinformation. Accessed 16 Oct 2020.

84. Liu Z, Song L, Zhao S, Huang J, Ma L, Zhang J, et al. Direct growth of graphene/hexagonal boron nitride stacked layers. Nano Lett. 2011;11(5):2032-7. https://doi.org/10.1021/nl200464j.

85. Gurram M, Omar S, Zihlmann S, Makk P, Li QC, Zhang YF, et al. Spin transport in two-layer-CVD-hBN/graphene/hBN heterostructures. Phys Rev B. 2018;97(4):045411.

86. Wang X, Li X, Zhang L, Yoon Y, Weber PK, Wang H, et al. Ndoping of graphene through electrothermal reactions with ammonia. Science. 2009;324(5928):768-71. https://doi.org/10.1126/ science.1170335.

87. Martins TB, Miwa RH, da Silva AJR, Fazzio A. Electronic and transport properties of boron-doped graphene nanoribbons. Phys Rev Lett. 2007;98(19):196803.

88. Albert D, Mahfuza K. Electronic properties of edge-terminated zigzag hexagonal boron nitride nanoribbons. J Phys D Appl Phys. 2019;52(2):025301

89. Mukherjee R, Bhowmick S. Edge stabilities of hexagonal boron nitride nanoribbons: a first-principles study. J Chem Theory Comput. 2011;7(3):720-4. https://doi.org/10.1021/ct1006345.

90. Park C-H, Louie SG. Energy gaps and stark effect in boron nitride nanoribbons. Nano Lett. 2008;8(8):2200-3. https://doi.org/10. 1021/n1080695i.

91. García-Miranda Ferrari A, Brownson DAC, Abo Dena AS, Foster CW, Rowley-Neale SJ, Banks CE. Tailoring the electrochemical properties of 2D-hBN via physical linear defects: physicochemical, computational and electrochemical characterisation. Nanoscale Adv. 2020;2(1):264-73. https://doi.org/10.1039/C9NA00530G.

Publisher's note Springer Nature remains neutral with regard to jurisdictional claims in published maps and institutional affiliations. 